\title{
Simplified and miniaturized laser Doppler distance sensor for dynamic position and shape measurement at rotating objects
}

\author{
Thorsten Pfister, Lars Büttner, Jürgen Czarske \\ Technische Universität Dresden, Institut für Grundlagen der Elektrotechnik und Elektronik, \\ Professur für Mess- und Prüftechnik, Helmholtzstraße 18, 01062 Dresden, Germany
}

\begin{abstract}
We present a simple and miniaturized laser Doppler distance sensor comprising only one single fanshaped interference fringe system for dynamic position and shape measurement of fast moving objects with micrometer precision. Due to its low complexity, it can be built very compact and cheap, which is crucial for many industrial applications. It will be shown theoretically as well as experimentally that its position uncertainty is in principle independent of the object velocity in contrast to conventional distance sensors. In order to evidence the operational capability and the application potential of this simplified laser Doppler distance sensor, radial and axial shape measurements of rotating bodies were carried out in comparison with a commercial triangulation sensor. An average postion resolution of $2.3 \mu \mathrm{m}$ was obtained.
\end{abstract}

\section{Introduction}

In-process measurement of position and shape as well as dynamic deformations and vibrations of fast moving and especially rotating objects, such as turning parts, gear shafts, rotors and turbine blades, is an important task both in production engineering and process control. However, this is a big challenge for metrology, since absolute and contactless measurement techniques with high position resolution and concurrently high temporal resolution are required.

Capacitive and inductive sensors are often not applicable due to electromagnetic disturbances, e.g. in the vicinity of electric motors. Optical measurement techniques overcome this drawback and also offer higher accuracy. However, incremental sensors such as Michelson interferometer or laser scanning vibrometer are not suitable, because their measurement results become ambiguous if distance jumps of more than half the laser wavelength occur. Furthermore, the measurement rate of most optical techniques is fundamentally limited either by the speed of mechanical scanning (time domain OCT, auto-focus sensing) or by the detector frame rate and minimum exposure time (triangulation, chromatic confocal sensing, Fourier domain OCT). Therefore, highly dynamic measurements are not possible with these sensors.

In order to cope with these problems, we recently developed a laser Doppler distance sensor (LDDS) ${ }^{1}$, which is an extended and modified laser Doppler velocimeter employing two superposed fan-shaped interference fringe systems with contrary fringe spacing gradients $[1,2]$. Via the resulting two independent measuring channels represented by these two fringe systems, two different measurands, i.e. axial position (distance) and tangential velocity of a moving object surface, can be determined simultaneously. Thus, the shape of rotating objects can be measured absolutely and with one single sensor only. The outstanding feature of this sensor is that its position uncertainty is in principle independent of the object velocity enabling precise, dynamic and in-process position and shape measurements on fast moving objects, such as rotating shafts, workpieces and turbo machine rotors [2-5].

In this contribution, we introduce a simplified version of the LDDS comprising only one single fan-shaped interference fringe system, which is named divergent fringe sensor (DFS) $[2,6]$. Due to its low complexity, it can be built very compact and cheap, which is crucial for many industrial applications. Nevertheless, this simplified sensor can also be applied to position and shape measurements of fast moving and especially rotating objects, provided that the tangential velocity of the object surface is known, which holds for many practical applications (see section 2). In the following, first the fundamental idea and the functional principle is explained, then, the measuring features and the operational capability of the DFS are investigated theoretically as well as experimentally and, finally, a miniaturized sensor prototype is presented employing fiber optics and only a minimum number of optical elements.

\footnotetext{
${ }^{1}$ In 2008, the Berthold Leibinger Innovationspreis (3rd prize) was awarded to the authors for inventing the LDDS.
} 


\section{Fundamental idea and functional principle}

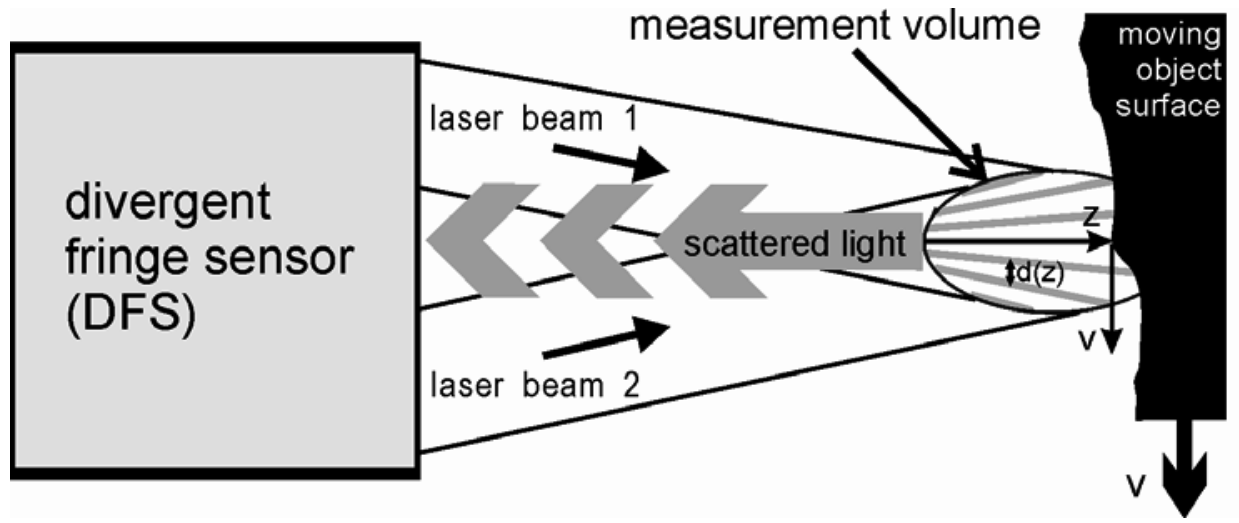

Fig. 1: Idea and application of the divergent fringe sensor (DFS).

With the LDDS, axial position $z$ and tangential velocity $v$ of a moving object surface, can be determined simultaneously via the two available measuring channels [1-3]. However, in practice, there are many applications, where the tangential velocity $v(t)$ of the object surface is known very well. This a priori knowledge of the tangential object velocity can be exploited for a simplification of the LDDS resulting in the novel DFS, which accordingly comprises only one measuring channel, i.e. only one fan-shaped interference fringe system, in contrast to the LDDS.

With a proper sensor setup (for details see $[2,6]$ ), the necessary fan-shaped interference fringe system with an one-to-one interrelation $d(z)$ between the fringe spacing $d$ and the axial object position $z$ can be generated in the crossection of two coherent laser beams (fig. 1). The Doppler frequency $f_{D}$ of the scattered light signal, which is detected in backward direction, is directly related to the transverse object velocity $v$ by $v=f_{D} \cdot d(z)$. The fringe spacing function $d(z)$ is approximately linear [6] and can be described as

$$
d(z)=d_{0}+m z
$$

with the slope $m=\partial d / \partial z$. Its inverse is given by

$$
z=z_{0}+m^{-1} d=z_{0}+m^{-1} \frac{v}{f_{D}} .
$$

The slope $m$ as well as the constants $d_{0}$ and $z_{0}$, which are related to each other by $z_{0}=-d_{0} / m$, are known from the calibration of the sensor. Thus, the axial position $z$ of a measurement object passing this fan-shaped fringe system with a known tangential velocity $v$ (see fig. 1) can be calculated absolutely and unambiguously via the measured Doppler frequency $f_{D}$ using equation (2). Consequently, the DFS enables absolute position and shape measurements on laterally moving objects with only one fan-shaped interference fringe system provided that the tangential object velocity is known.

This condition is fulfilled in particular for many applications at rotating objects [6]. For example at machine tools, motors or turbo machines, mostly, the current rotational frequency $f_{\text {rot }}(t)$ is continuously measured by speed sensors inside the machine. Alternatively, $f_{\text {rot }}(t)$ can be determined directly from the laser Doppler measurement signals via a proper spectral analysis [1]. If the object radius $R$ is also known, the tangential velocity at the object surface can be calculated precisely according to

$$
v(t)=2 \pi f_{\text {rot }}(t) R \text {. }
$$

In this context, two major application areas concerning rotating objects can be distinguished, where the object radius and, thus, the tangential velocity is known (see also section 4 , fig. 3 ):

(I) The first area comprises radial measurements of radius variations $\Delta z(\varphi)=\Delta R(\varphi)$ (e.g. due to unbalances and deformations) and of the resulting two-dimensional shape $R(\varphi)=\bar{R}+\Delta R(\varphi)$ of rotating objects against the rotation angle $\varphi$. For instance at big rollers as well as at large rotors of motors and turbo machines with a mean radius in the range of $\bar{R}=(0.1 \ldots 1) \mathrm{m}$, the radius variations, which have to be measured, usually do not exceed $\Delta R=(0.1 \ldots 1) \mathrm{mm}$. At fast rotating shafts with a radius in the magnitude of $1 \mathrm{~cm}$, the amplitude of unbalances and vibrations typically amounts to only a few micrometers. For all these applications, the mean object radius $\bar{R}$ is generally known and the maximum relative radius variation does not exceed $|\Delta R / \bar{R}|=10^{-3}$. Consequently, the 
condition $|\Delta R(\varphi)|<<\bar{R}$ is fulfilled and, thus, the tangential object velocity is known very well from equation (3) by inserting $R \approx \bar{R}$.

(II) The second application area is axial measurements of position, shape, thickness variations, displacement as well as vibrations of rotating and laterally moving objects with known tangential velocity (see fig. 3, right). E. g. in turbo machinery, the characterization of axial rotor oscillations is an eminent task in order to achieve an optimized and save operation. In this case, the radius $R=\bar{R}$ is constant and there are no radius variations. If this radius $R$ is known with high accuracy, the tangential velocity $v$ can be determined precisely according to equation (3).

Consequently, in both cases, the condition for applying the DFS is fulfilled. Fig. 2 summarizes the functional principle of the DFS once again in block diagram form.

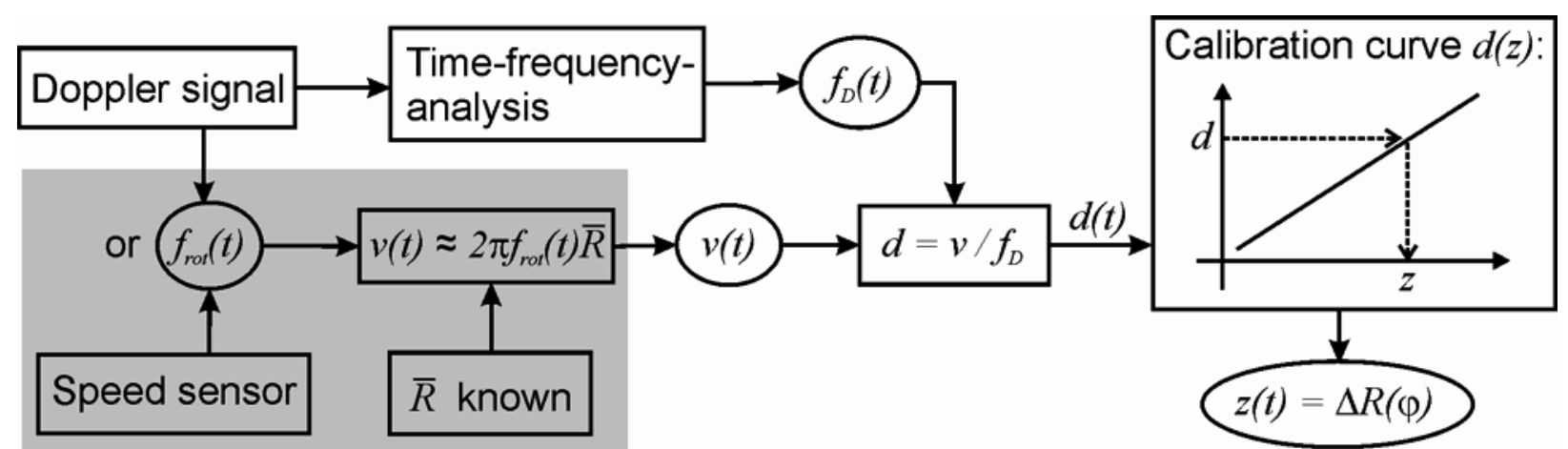

Fig. 2: Block diagram depicting the functional principle of the DFS $[2,6]$. The gray box accounts for position and shape measurements at rotating objects.

\section{Measurement uncertainty}

The achievable position resolution $\sigma_{\mathrm{z}}$ of the DFS, which is defined as the average statistical error constituting the minimum obtainable measurement uncertainty, can be derived analytically from equation (2) using the law of error propagation resulting in [6]:

$$
\sigma_{z} \approx \frac{\bar{d}}{m} \frac{\sigma_{f_{D}}}{f_{D}} .
$$

Thus, the position resolution depends on the slope $m$ of the fringe spacing curve, on the average fringe spacing $\bar{d}$ and on the relative measurement uncertainty of the Doppler frequency $\sigma_{f_{D}} / f_{D}$. Inserting the Cramer-Rao lower bound for the frequency measuring error of noisy single-tone signals into equation (5), it can be rewritten as [6]

$$
\sigma_{z} \approx \frac{\sqrt{3}}{\pi} \frac{\bar{d}^{2}}{m \Delta x \sqrt{S N R} \sqrt{N}}
$$

Consequently, the position resolution $\sigma_{z}$ depends, besides the slope $m$ of the fringe spacing curve and the average fringe spacing $\bar{d}$, on the averaging length on the object surface $\Delta x$, on the signal-to-noise ration SNR of the measured scattered light signals and on the number $N$ of statistically independent samples recorded within the measuring time $\Delta t=\Delta x / v$. However, $\sigma_{z}$ is independent of the object velocity $v$ similarly to the LDDS [4]. Therefore, the DFS is capable of carrying out precise position and shape measurements at arbitrarily fast moving objects, such as turbo machine rotors.

In the case of radial shape measurements on rotating objects (see section 2, application area (I)), additional systematic position errors $\Delta_{z}$ may occur due to uncertainties with regard to the object velocity. If measurement errors in the rotation frequency $f_{r o t}$ as well as calibration errors can be neglected, this additional systematic position error is given by

$$
\Delta_{z}=\frac{\bar{d}}{m} \frac{\Delta v}{v} \approx \frac{\bar{d}}{m} \frac{\Delta R}{R} .
$$

The sensor setup used for all the measurements described in this paper exhibited a fringe spacing slope of $m=1.82 \times 10^{-3}$ and an average fringe spacing of $\bar{d}=4.6 \mu \mathrm{m}$. Depending on the SNR of the detected 
measurement signals, the relative uncertainty of the Doppler frequency is typically in the range of $\sigma_{f_{D}} / f_{D} \approx(1 \ldots 10) \times 10^{-4}$ [2]. Thus, the position resolution of the DFS sensor setup used can be estimated to $(0.3 \ldots 2.5) \mu \mathrm{m}$ using equation (4). The additional systematic position error due to uncertainties with regard to the object velocity, which occurs at radial shape measurements on rotating objects (section 2 , application area (I)), amounts to $\Delta_{z} \leq 2.5 \mu \mathrm{m}$ assuming that the relative radius variation does not exceed $|\Delta R / \bar{R}|=10^{-3}$. Consequently, an overall standard uncertainty for the position measurement below $3 \mu \mathrm{m}$ can be obtained theoretically even for radial position and shape measurements on rotating objects.

\section{Experimental results of radial and axial shape measurements at rotating bodies}

In order to demonstrate the operational capability of the DFS as well as its potential for practical applications, test measurements corresponding to the two different application areas concerning rotating objects specified in section 2 were carried out in comparison with a commercial triangulation sensor (see fig. 3). The applied triangulation sensor exhibits a position resolution of $1 \mu \mathrm{m}$ and a linearity of $\pm 8 \mu \mathrm{m}$ at the maximum sampling rate of $2.5 \mathrm{kHz}$. Both objects were driven by a speed-controlled electric motor, whose rotational frequency was measured continuously and precisely with an optical speed sensor.

The $3 \mathrm{~mm}$ thick brazen disk employed for the radial shape measurement experiments (fig. 3 , left) exhibited a mean radius of $\bar{R}=42.93 \mathrm{~mm}$ and a radius variation along its circumference of $\pm 90 \mu \mathrm{m}$. Accoring to equation (6), the resulting maximum systematic measurement error yields $\Delta_{z}=5.0 \mu \mathrm{m}$. The test object used for the axial shape measurements (fig. 3, right) consisted of four sheet steels of $150 \mu \mathrm{m}$ thickness, which were clamped onto a rotating base plate. The individual sheets were shaped in a way that, in axial direction, a cascaded height profile consisting of three steps of $150 \mu \mathrm{m}$ was formed along the circumference of the object. Here, the measuring position for both sensors was located at a constant radial distance to the rotation axis of $R=28.5 \mathrm{~mm}$ so that systematic errors due to a velocity uncertainty are excluded. For all measurements, the rotational frequency was $20 \mathrm{~Hz}$ and the lateral averaging length for measurement data evaluation was set to $\Delta x=2 \mathrm{~mm}$ for both sensors. Due to the limited sampling rate of the triangulation sensor, a smaller averaging length was not possible.

A comparison between the measurement results of both sensors is shown in fig. 4. In general, the radius profiles and the height profiles, respectively, obtained with the two different sensors match very well. Especially the measured radius and height jumps respectively at certain angular positions are equal and clearly visible in all measurement curves. The average measurement deviations between the results of the DFS and of the triangulation sensor are about the same for both experiments and amount to $7.5 \mu \mathrm{m}$ for the radial shape measurment (fig. 4, left) and to $11.0 \mu \mathrm{m}$ for the axial height profile measurment (fig. 4 , right) [6]. These deviations are caused by fundamental differences between the mesurement principles of both sensors, by their inherent measurement uncertainties as well as by not negligible differences in their measuring positions which were unavoidable in practice. Especially the additional systematic position error at the radial shape measurement due to the uncertainties with regard to the object velocity could not be detected, because it was too small compared to other interferences.
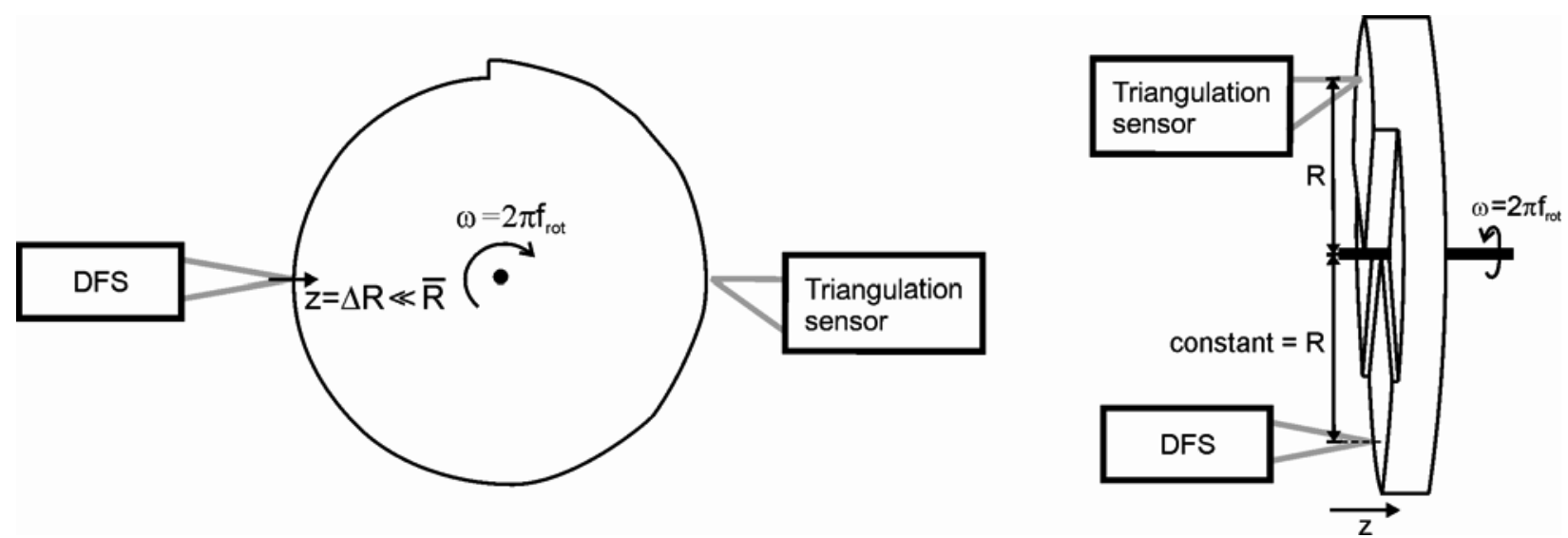

Fig. 3: Experimental setups according to the two different application areas concerning rotating objects described in section 2. Left: radial shape measurement of a rotating brazen disk exhibiting relatively small radius variations. Right: axial shape measurement of a rotating body exhibiting a height profile with several axial steps. In both cases, a commercial triangulation sensor was used as a reference $[2,6]$. 

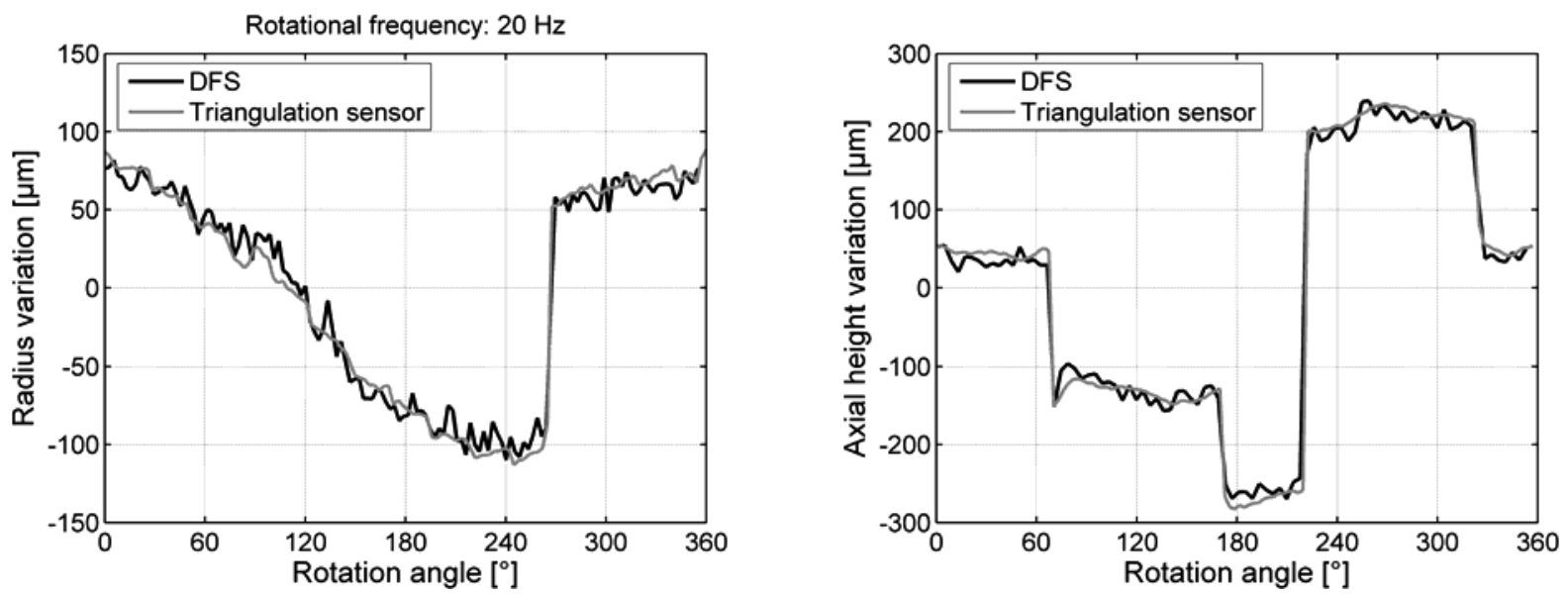

Fig. 4: Circumferential radius radius variations (left) and axial height profile (right) measured with the DFS compared to the measurement results of the triangulation sensor corresponding to the two experimental setups from fig. 3 [6].

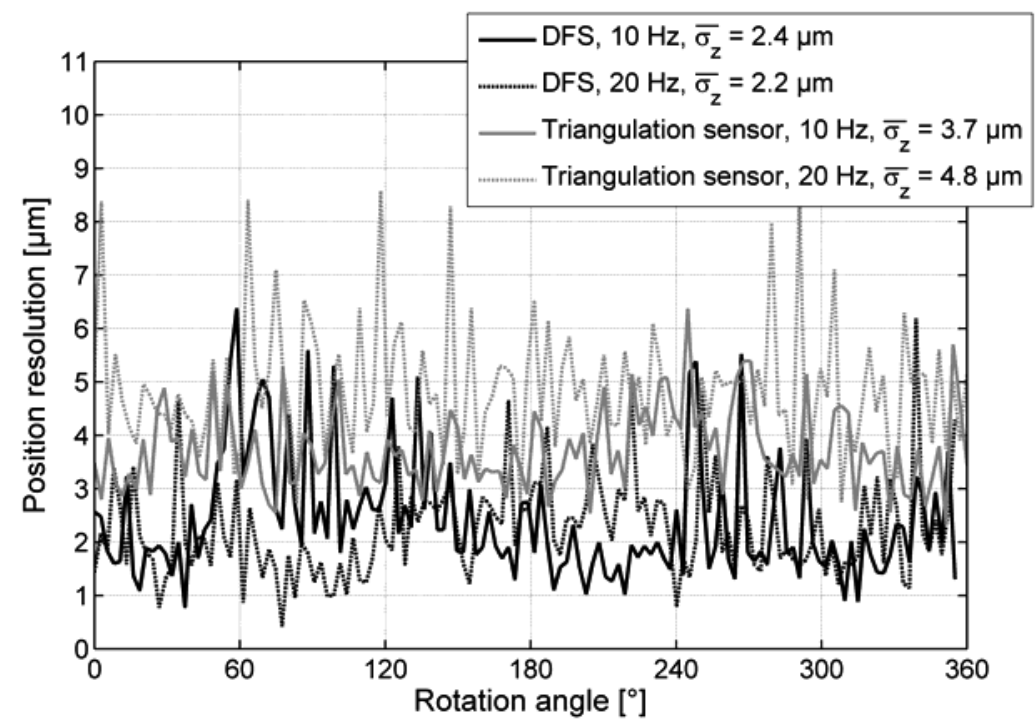

Fig. 5: Measured position resolution $\sigma_{z}$ of the DFS and of the triangulation sensor at the radial shape measurment and at two different rotational frequencies of $10 \mathrm{~Hz}$ and $20 \mathrm{~Hz}$, respectively, depicted as a function of the rotation angle [6].

In fig. 5, the standard deviations $\sigma_{z}$ of the measured radial positions representing the position resolutions of both sensors are depicted as a function of the rotation angle for the radial shape measurement and for two different rotational frequencies of $10 \mathrm{~Hz}$ and $20 \mathrm{~Hz}$. For the triangulation sensor, the average standard deviation increases considerably from $3.7 \mu \mathrm{m}$ to $4.8 \mu \mathrm{m}$ along with the increase of the rotational frequency from $10 \mathrm{~Hz}$ to $20 \mathrm{~Hz}$. In contrast, the average position resolution of the DFS remains constant at about $2.3 \mu \mathrm{m}$ independent of the rotational frequency (see fig. 5). This is in perfect agreement with the theoretical considerations in section 3 and it confirms that, in contrast to conventional distance sensors such as triangulation sensors, the position resolution of the DFS is in principle independent of the object velocity in analogy to the LDDS $[4,6]$.

\section{Miniaturized sensor prototype}

The complexity of the setup of the DFS is considerably reduced compared to the LDDS since one single fan-shaped interference fringe system suffices [2,6]. Therefore, only one laser light source and one photo detector but no multiplexing elements are required. Also, the effort for data acquisition and signal processing is significantly lower, because only one measurement signal has to be acquired and evaluated. Consequently, the DFS can be realized much cheaper and much more compact than the LDDS.

Fig. 6 shows a schematic diagram as well as pictures of a first miniatur DFS prototype employing fiber optics and only a minimum number of optical elements. The laser beam guided via a singlemode fiber 
(SMF) to the sensor head is split by a transmission grating, whose +1 . and -1 . diffraction orders are brought to an intersection by a single lens generating the measurement volume containing a fan-shaped interference fringe system. The light scattered backward from the measurement object is coupled into a multimode fiber (MMF) by two $45^{\circ}$ mirrors and guided to a photo detector, which is connected to an A/D converter and a subsequent computer for signal evaluation. The overall size of the sensor head is only $55 \mathrm{~mm} \times 33 \mathrm{~mm} \times 35 \mathrm{~mm}$. In addition, it ca be built relatively inexpenively and, thus, it is suitable for broad industrial application.
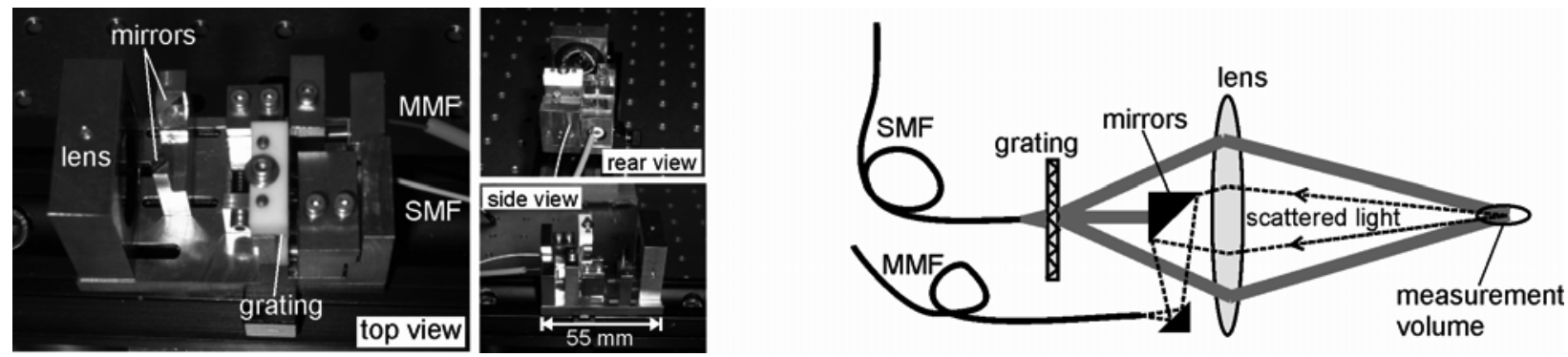

Fig. 6: Pictures for different views (left) and schematic diagram (right) of the sensor head of the miniaturized DFS prototype.

\section{Conclusions}

A simplified laser Doppler distance sensor comprising only one single fan-shaped interference fringe system was developed for dynamic position and shape measurement of fast moving and especially rotating objects, whose tangential velocity is known, which holds for many practical applications. It was shown theoretically as well as experimentally that the position uncertainty of this simplified DFS is in principle independent of the object velocity in analogy to the standard LDDS and in contrast to conventional distance sensors. Radial and axial shape measurements of rotating bodies were carried out in comparison with a commercial triangulation sensor evidencing the operational capability and the application potential of the DFS, for which an average postion resolution of $2.3 \mu \mathrm{m}$ was obtained. In addition, a first miniaturized sensor prototype was presented demonstrating that the DFS can be built very compact and low-cost due to its low complexity, which is crucial for many industrial applications.

\section{Acknowledgements}

The authors thank $P$. Klotsche for his excellent experimental work. Furthermore, the financial support of the Deutsche Forschungsgemeinschaft (DFG, project number: $C z$ 55/19-1) is greatly acknowledged.

\section{References}

[1] T. Pfister, L. Büttner, J. Czarske, „Laser Doppler profile sensor with sub-micrometre position resolution for velocity and absolute radius measurements of rotating objects“, Meas. Sci. Technol. 16, 627-641, 2005.

[2] T. Pfister, „Untersuchung neuartiger Laser-Doppler-Verfahren zur Positions- und Formvermessung bewegter Festkörperoberflächen“, Shaker Verlag, Aachen, 2008.

[3] T. Pfister, L. Büttner, J. Czarske, „In-process shape and roundness measurements at turning machines using a novel laser Doppler profile sensor", 8th Int. Symposium on Laser Metrology (Merida, Mexico, 14-18 February 2005), Proc. SPIE 5774, 44-55, 2005.

[4] T. Pfister, L. Büttner, J. Czarske, H. Krain, R. Schodl, „Turbo machine tip clearance and vibration measurements using a fibre optic laser Doppler position sensor", Meas. Sci. Technol. 17, 1693-1705, 2006.

[5] L. Büttner, T. Pfister, J. Czarske, „Fiber optic laser Doppler turbine tip clearance probe“, Optics Letters 31, 1217-1219, 2006.

[6] T. Pfister, L. Büttner, J. Czarske, „Laser Doppler sensor employing a single fan-shaped interference fringe system for distance and shape measurement of laterally moving objects“, Applied Optics 48, 140-154, 2009. 